\title{
A proposito di alcune mostre: le canzoni italiane e il Premio Cremona
}

\author{
di Adolfo Mignemi
}

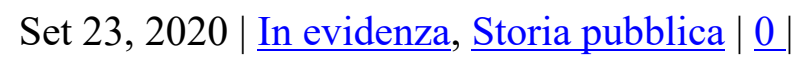

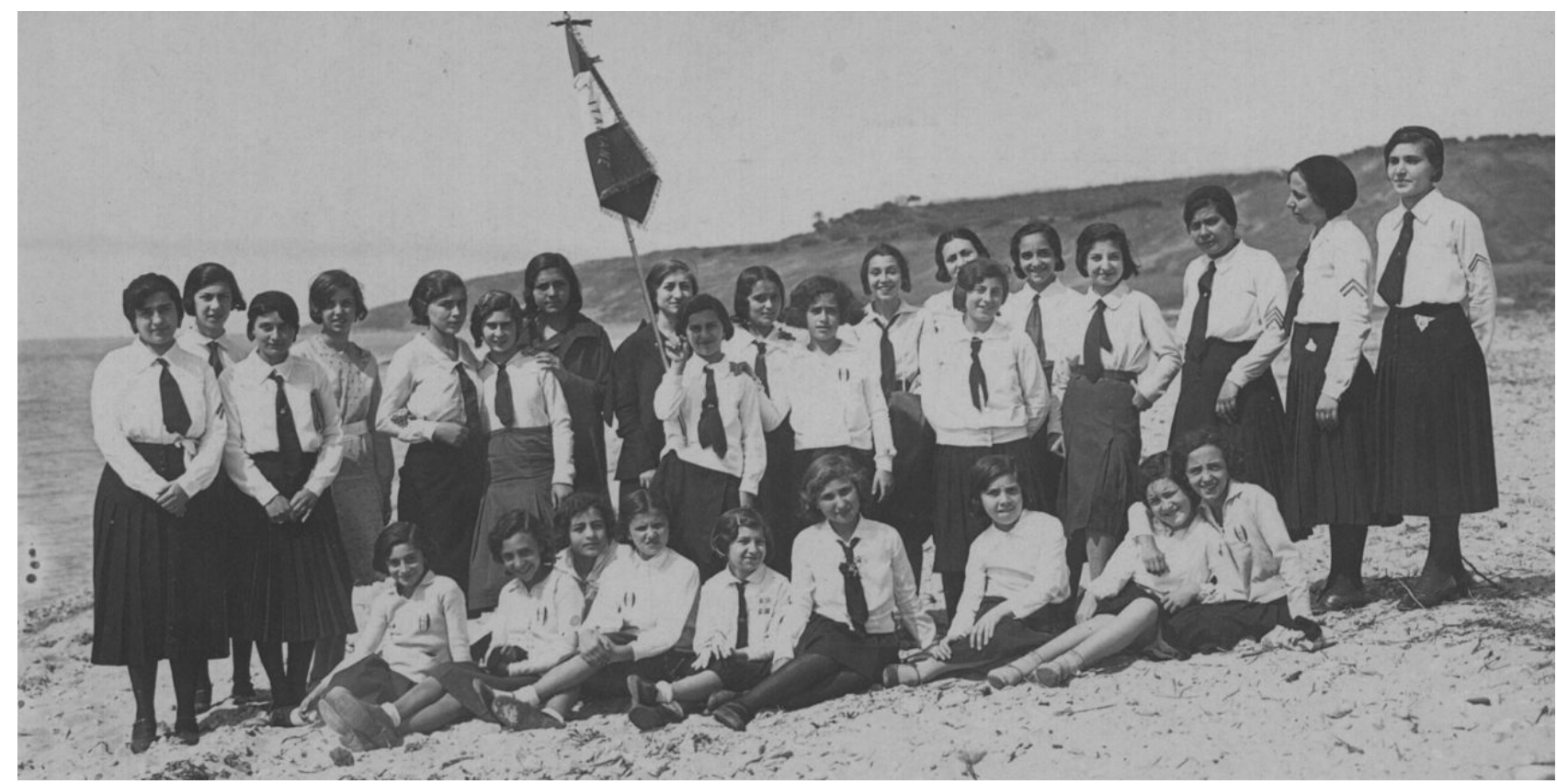

Le "piccole italiane"

By Unknown author - Archivio di Famiglia, CC BY-SA 3.0, Link

\begin{abstract}
I mesi di sospensione delle attività culturali in presenza hanno offerto l'occasione di riflettere e fare il punto su alcune esperienze tenutesi nei mesi precedenti.

In una riflessione in due puntate, lo storico Adolfo Mignemi ci conduce attraverso quattro mostre svoltesi negli ultimi due anni nel nostro Paese, ragionando sul valore della contestualizzazione storica e su problemi e opportunità offerti da oggetti che non sempre trovano spazio nei percorsi espositivi e museali.
\end{abstract}

\section{Mettere in mostra le nuove fonti}

La riflessione sulle nuove fonti, che si sono rese disponibili al lavoro dello storico o che nell'ultimo secolo sono state riscoperte in quanto tali, è in costante evoluzione. Pensiamo, da un lato, alle immagini e ai suoni fissati meccanicamente oppure agli oggetti, dall'altro alle produzioni artistiche, ai monumenti, alle città, ai paesaggi antropizzati, ai rituali ecc. Questo percorso avviene di solito tra sensazioni di entusiasmo, ma al tempo stesso di sgomento, in un impeto cogente e inderogabile, che impone di riprendere ed approfondire i temi della scrittura storiografica. Perché? Perché, come era accaduto per le fonti scritte quando lo storico moderno recepì la necessità di renderle accessibili, oggi gli strumenti di cui disponiamo per comunicare e divulgare ci sollecitano ad agire di conseguenza con le nuove fonti: le immagini devono poter essere viste da tutti, gli oggetti devono poter essere mostrati, i suoni devono poter essere ascoltati. Ecco, appunto: devono poter rientrare 
nella narrazione come siamo stati abituati a fare con le parole scritte. Ma siamo effettivamente all'altezza di questo compito? È veramente più facile e più accessibile l'atto di mettere in mostra questi documenti rispetto al tradizionale e ormai abituale allineare su di un foglio, nella scrittura, soggetti verbi aggettivi avverbi e raccordarli a nomi e date? Ogni volta che visitiamo una mostra o un museo ci poniamo queste domande ma troppe volte ci rispondiamo che quelle narrazioni sono sempre inadeguate, che la scrittura pecca di ingenuità capaci di generare più confusione che chiarezza, di travisare piuttosto che spiegare adeguatamente attraverso le forme più comuni di conoscenza di cui disponiamo.

\section{Documenti tra emozione e informazione}

Perché le nuove fonti di cui lo storico di oggi ambirebbe appropriarsi altro non sono che gli oggetti, i suoni e le immagini che riemergono dal passato, in forma frammentaria e sempre incompleta, a documentare quanto accaduto, a testimoniare come si sono vissute determinate esperienze. E si tratta sempre di testimonianze che traboccano di elementi conoscitivi sempre più carichi di emozioni ma, al tempo stesso, privi di quella parte di informazioni che ogni documento non è stato in grado di registrare pienamente e che finiscono per risultare di fatto "obliate". La narrazione storiografica si è dovuta adeguare ai caratteri formali delle sue fonti fin dal principio, quando la reiterazione della memoria non riusciva ancora a strutturarsi nella comunicazione verbale scritta, a passare cioè dal suono alla scrittura. Ed è solo quest'ultima infatti che ha consentito fino al secolo scorso il diffondersi delle elaborazioni critiche degli eventi. A partire dal Novecento l'irruzione di fonti del tutto nuove come la registrazione meccanica delle immagini fisse (fotografie) e in movimento (cinema) nonché dei suoni, ha imposto una straordinaria trasformazione della narrazione storica ed altresì della loro critica. Accanto al nascere di una storiografia che riguardava i singoli mezzi e i conseguenti linguaggi comunicativi oggi si sta faticosamente affermando (sarà un processo lungo) anche una storiografia generale capace di rapportarsi alla natura di questi diversi tipi nuovi di fonti.

\section{Le mostre in esame}

Queste sintetiche considerazioni generali ci sembrano un indispensabile preambolo alle note relative a quattro mostre allestite negli ultimi due anni, tre centrate sull'epoca fascista e una che si propone di valorizzare una fonte raramente fatta oggetto di percorsi espositivi e che ci hanno condotto, nel bene e nel male, ad approfondire le tematiche sopra accennate. Le mostre sono, in ordine cronologico: Post Zang Tumb Tuuum Art Life Politics Italia 1918-1943, tenutasi a Milano dal 18 febbraio al 25 giugno 2018; Si faccia un articolo di fondo... 'Il Regime Fascista', Farinacci e il Ventennio a Cremona, allestita in questa stessa città dal 12 maggio al 30 settembre 2018, poi prorogata fino al 6 novembre e, sempre a Cremona, Il Regime dell'Arte. Premio Cremona 19391941, proposta dal 21 settembre 2018 al 24 febbraio 2019; infine Noi ... non erano solo canzonette, aperta a Torino tra il 22 marzo e il 7 luglio 2019 e successivamente riproposta a Bologna dal 29 novembre al 12 aprile 2020. Traendo spunto dalle note appuntate durante le visite ai percorsi espositivi e dalla lettura dei rispettivi cataloghi cercheremo di evidenziare, anche ponendo in contrapposizione i diversi percorsi, le numerose aspettative riposte in ognuna, le perplessità e gli aspetti difficilmente condivisibili di ognuna, non ultimo la preziosa riscoperta delle ineguagliabili potenzialità che l'uso associato delle fonti visive, sonore e scritte e il contatto diretto con esse può generare.

\section{Non solo canzonette}

La nostra "rilettura" prende come punto di partenza la visita alla bella mostra, Noi ... non erano solo canzonette, dedicata alle canzoni che hanno accompagnato la storia italiana tra il $1958 \mathrm{e}$ il1982, dalla quale era possibile uscire - impressione condivisa da vari altri visitatori - con la 
rafforzata convinzione che "la musica di uno specifico periodo storico - come hanno scritto i curatori - va di pari passo con la letteratura, l'arte, la politica, l'architettura, la cultura generale di quel tempo e una canzone, non meno di un libro o di un dipinto, sa riflettere il momento storico in cui è stata immaginata, scritta e cantata".[1] In altre parole che anche attraverso le cosiddette canzonette è possibile effettuare una corretta lettura, dal punto di vista storico, delle trasformazioni sociologiche ed economiche che hanno caratterizzato vari decenni di vita italiana, in questo caso specifico dapprima la "grande trasformazione" che "investì allora la società italiana scardinando vecchie abitudini, alimentando un falò in cui bruciarono comportamenti, appartenenze, identità, ribaltando tradizioni e consuetudini. In soli dieci anni da Paese contadino l'Italia diventò una delle massime potenze industriali del mondo, specchiandosi nei successi di una ricostruzione 'miracolosa' che aveva di colpo riscattato un lungo periodo di miseria, di dittatura, di guerra". Poi ci fu la "vicenda dolorosa e drammatica" del terrorismo e delle stragi "che pure non riuscì a scalfire i capisaldi della nostra democrazia che, anzi, proprio allora fece segnare un significativo allargamento dei diritti civili e della sfera inclusiva dello spazio pubblico". Infine vennero gli anni in cui "sulle macerie dell'utopia ugualitaria e collettiva degli anni settanta, si accamparono una vorace voglia di consumi e un nuovo stile di vita, fondato più sull'egoismo che sulla solidarietà".[2] Contribuivano allo spessore analitico della mostra una accurata indagine sia dal punto di vista linguistico dei testi cantati sia una ricostruzione filologica dei brani musicali tesa a ricostruire la versione più aderente a quella originale, sia, infine, il ricorso a una accorta selezione di immagini fotografiche di agenzia sui cantanti, con il compito di evocare anche il "consumo" collettivo di quel fenomeno che fu la diffusione della musica leggera in quell' arco cronologico.

\section{La contestualizzazione storica dei consumi musicali}

Anche se l'oggetto della narrazione storiografica perseguita dalla mostra erano i gusti musicali e l'evoluzione tecnologica dei "consumi" sonori, i curatori non si sono sottratti alla necessità di indagare i diversi processi di modernizzazione, sia dal punto di vista della vita quotidiana, sia dei rapporti produttivi, oltre ai processi di interazione culturale messi in atto dai fenomeni di migrazioni interne di carattere epocale, l'evoluzione delle sensibilità e dei comportamenti politici di massa e, non ultimi, l'evoluzione della lingua parlata e gli slittamenti semantici. Il percorso è stato progettato con intelligenza e grande conoscenza dei paralleli processi in atto nella società, nel mondo del lavoro, nelle modalità di esercitare i diritti e i doveri e nelle forme assunte dalle organizzazioni politiche passando attraverso l'esperienza, che il visitatore poteva fare (senza alcun onere supplementare!), di una comunicazione orale parallela e diretta fornita da un'audioguida caratterizzata dalla completezza e dalla costante possibilità di interromperla in qualsiasi momento per poter eventualmente riprenderla oppure passare direttamente alla narrazione successiva. Il testo narrante dell'audioguida consentiva un validissimo raccordo tra i diversi tempi della visita individuale e quelli offerti dal percorso espositivo. Tale testo, infatti, alternava il racconto degli eventi alle "citazioni" dei cento brani selezionati lasciando a sua volta ai vari "punti di ascolto" il compito di accedere alle "edizioni critiche" delle canzoni poste al centro delle dodici aree tematiche in cui la mostra era articolata. Altro aspetto fondamentale che la visita poneva in evidenza era il fatto che alla base della progettazione e dell'allestimento vi fosse stata l'attività di un gruppo di lavoro che si era imposto di disporre sempre, al suo interno, di risorse sul piano storico capaci di raccordare la fruizione dei brani musicali a una sintetica ma precisa restituzione delle dinamiche degli eventi che ne avevano accompagnato la diffusione e il progressivo affermarsi come forma tra le più significative di rappresentazione collettiva.

\section{Il Premio Cremona 1939-1941}

Questo ultimo aspetto è esattamente ciò che non si manifestava nella mostra dedicata al "Premio Cremona", dal titolo Il Regime dell'Arte. Premio Cremona 1939-1941. Ci è capitato di visitarla pochi giorni dopo l'apertura quindi, in teoria, nella situazione di massima efficienza di tutte le 
strutture espositive e di ogni dispositivo predisposto per rendere più coinvolgente il percorso di visita. La mostra ("mai prima allestita", hanno scritto i curatori[3]) ripercorreva la vicenda del “'Premio Cremona', il concorso pittorico voluto da Roberto Farinacci nel 1939 con l'intento di sostenere l'idea dell'arte come celebrazione dei valori e delle imprese del fascismo". Il concorso fu rilanciato nei due anni successivi e si progettò una quarta edizione nel 1943 che non ebbe mai luogo. A differenza della mostra di Torino sulla musica leggera in questo caso il visitatore era accolto da informazioni generali estremamente succinte e da una parete che raccoglieva forse tutti i modelli messi in commercio della "Radio Balilla". Gli apparecchi riproducevano all'unisono l'annuncio radiofonico diffuso dall'Eiar delle notizie propagandistiche relative al "Premio Cremona". Un metodo veramente singolare questo utilizzato dai curatori per mettere in evidenza, si può presumere, la natura e gli strumenti della propaganda fascista! La parete con le "Radio Balilla" era corredata da un testo di ventisette righe di cui diciotto erano impegnate dalla, pur doverosa, descrizione tecnica dei materiali. Nel restante testo si è ritenuto di esaurire la questione del ruolo della radiofonia nel sistema delle comunicazioni di massa gestite e controllate dal regime fascista. Non una sola parola è stata invece spesa sulle peculiarità del mezzo, sull'originalità dei modi in cui esso fu utilizzato, ad esempio, per costruire la fisicità della voce di Mussolini con la speranza, forse, che la forza esplicativa dei quadri dell'edizione 1939 del Premio - accomunati dal tema unificatore "Ascoltazione del discorso del duce alla radio" - fosse sufficiente. Gli unici testi di guida alla visita erano le indicazioni autoriali dei materiali esposti e qualche sporadica annotazione di carattere storico-artistico. Il visitatore si trovava come paracadutato tra quadri, bozzetti e prove dei quadri presentati alle tre edizioni del Premio senza poter disporre di alcuna indicazione utile né sul contesto in cui il Premio era nato né sulla sua vicenda. Nulla che approfondisse il contesto storico generale (dalla seconda edizione del premio in poi si era ormai in piena seconda guerra mondiale) e delineasse la dimensione puramente propagandistica del Premio, nonché la strumentalità dei temi. I testi illustrativi erano costituiti da tre lunghe frasi. La prima di Vittorio Sgarbi, curatore con Rodolfo Bona della mostra, che l'ha voluta firmare nello stile delle citazioni mussoliniane dipinte sui muri delle città italiane durante il ventennio di dittatura. La scritta era posta a sovrastare la parete che ospitava il quadro di Giuseppe Moroni, "Colonie fluviali". Su tale scritta ritorneremo più avanti. La seconda frase era una citazione di Roberto Farinacci: "Questo novecentismo fascista: forte, vigoroso, epico, romano". Essa dominava la parete dedicata alla "Radio Balilla". La terza, infine, era di Bona che non l'ha voluta firmare ed era posta a sovrastare il trittico di Baldassarre Longoni dedicato alla "Battaglia del grano".

\section{Una mostra senza "pentimento e vergogna"}

Eppure la mostra era presentata come una iniziativa carica di grandi ambizioni: "L'iniziativa cremonese consente, in modo sofisticato e imprevedibile, di ricostruire un preciso periodo storico con l'intento di riabilitare un'arte ripudiata e bistrattata, ritenuta impresentabile e indegna di attenzione, vittima di un pregiudizio inevitabilmente condizionato da quello negativo sul fascismo".44] Così aveva dichiarato Sgarbi: "con questa presenza aliena da ogni esaltazione del fascismo, e invece racconto di momenti di vita, Il Premio Cremona rientra nella storia di alcuni momenti essenziali della nostra vita civile di quegli anni, raccontati da maestri che erano stati dimenticati o rimossi per un grave peccato di presunzione della critica. Oggi Cremona ripara quella ferita".[5] Addirittura i manifesti di promozione commerciale dell'esposizione sotto il ritratto del critico, ne riproducevano l'ennesima precisazione: "Occorreva coraggio e una visione rispettosamente storicistica per recuperare le tele divise fra Cremona e Hannover, e ricostruire lo spirito e l'aria dei tempi. Quelli in cui i miei genitori furono Balilla e Giovane Italiana. Perché vergognarsi di ciò che è stato? Perché nascondere i gruppi familiari in ascolto dei discorsi del Duce alla radio?" La vera risposta era nell'affermazione che seguiva e concludeva il testo: "Per la prima volta, una mostra sull'arte del fascismo non protesta pentimento e vergogna, ma ci mette davanti a quello che è stato". Si è accennato alla prima delle tre citazioni-guida della mostra. In essa si 
presentava il quadro di Moroni con questa frase: "Moroni pettina armoniosamente, con gruppi ordinati di giovani educati e mansueti, senza distinzioni sociali, una bionda spiaggia su un'ansa del Po, in prossimità di Cremona. Ed è beatitudine terrena, in lidi domestici”. È di certo anche questo un "modo" di leggere la storia. La rappresentazione del processo di militarizzazione di una nazione in tutte le fasce d'età dei cittadini può essere letta in tutta serenità anche se quegli stessi giovani della GIL raffigurati da Moroni avrebbero marciato, a distanza di pochi mesi, nell'estate del 1940, unendosi ad altri ventiduemila provenienti da varie regioni italiane, percorrendo in lungo e in largo la pianura padana e accumulando 420 chilometri, alla volta di Padova, dove "la marcia della giovinezza" venne accolta, il 10 ottobre, da Mussolini che promise ai giovani partecipanti l'immediata "prova del fuoco" sul campo di battaglia; salvo poi, fortunatamente, un mese dopo rispedirli tutti a casa "per dedicarsi studio e lavoro durante prossima stagione invernale".

\section{L’artista può essere senza essere del proprio tempo?}

D'altro canto, sottolineava il catalogo, ricorrendo all'ennesima interrogazione retorica: l'artista "può essere senza essere del proprio tempo?".6] A questo punto però vorremmo proprio capire di quale "essere del proprio tempo" si intendesse parlare. Evidentemente delle piroette disinvolte che di fronte ai cambiamenti politici epocali vedono spesso alcuni intellettuali e artisti esibirsi in repentini e radicali trasformismi. Perché i curatori non ignoravano di certo che proprio nel quadro di cui stiamo parlando, "al centro del dipinto si impone il militaresco quadrato, con le figure maschili legnose e sull'attenti, allineate e ordinate, inquadrate per angolo sotto uno svettante tricolore e che, originariamente, tendevano il braccio nel saluto romano".[7] Cosa vuol dire tutto questo? Che Moroni a fine guerra, per rendere commerciale la tela, la privò di ogni connotazione politica cancellando il saluto romano e, molto probabilmente, anche l'emblema sabaudo dal campo bianco della bandiera nazionale? La cosa appare più che probabile. Ma ad affermare ad oltranza, senza essere sfiorato da alcun dubbio, la totale innocenza/onestà intellettuale dell'artista, in qualsiasi tempo e luogo, nel contesto della mostra sul "Premio Cremona" di cui ci si stava occupando, era Bona che aveva scelto il trittico di Baldassarre Longoni per esemplificare - ed era il secondo grande slogan-testo proposto dalla mostra - come "contro chi cerca di piegare l'arte alle proprie finalità, con ogni sua pennellata il pittore innalza la bandiera colorata della libertà dello spirito". I percorsi artistici sono prodotti storici che trovano le proprie radici nel passato oltre che nel presente. Essi sono tutt'altro che avulsi dai contesti in cui l'artista opera e non possono invocare nessuna autonomia che li ponga al di sopra delle parti. Gli artisti che dipingevano o scolpivano per il regime, così come coloro che operavano contro, al di là della tecnica e dei metodi espressivi, della ricerca formale, vanno studiati nel contesto storico in cui hanno agito, per le opere che in esso hanno prodotto, per la committenza che ne è stata all'origine e per le ricadute che il loro lavoro e la loro ricerca hanno avuto all'interno di quel contesto. Questo approfondimento è doveroso e imprescindibile per chi è vissuto in un'epoca, quale appunto il XX secolo, in cui la politica ha arruolato tutte le forme di comunicazione facendone una vera e propria arma: il "Premio Cremona" fu uno dei momenti più significativi della vicenda nazionale italiana negli anni del regime fascista. Per questo va studiato ma nella correttezza. Qui invece siamo di fronte al tentativo di legittimare un approccio anti-storiografico che nella rimozione perseguita e voluta ad ogni costo trova la giustificazione per affrontare senza "pentimenti e vergogna" l'analisi della produzione artistica di un preciso momento storico. A parte che pentimento e vergogna corrispondono solitamente a un atteggiamento tutto soggettivo generato da un comportamento individuale e quindi questa emotività riguarda unicamente Sgarbi e Bona, ma di fronte al fascismo e alla sua penetrazione "culturale" tra gli italiani, storicamente è ormai da anni acquisito che essa non fu un'infausta anomalia, bensì il punto d'arrivo di un percorso delle contraddizioni politiche, sociali e culturali che il Paese portava avanti da decenni.

\section{Testi senza contesto}


Qual è l'interesse di mettere in mostra dei quadri del Premio Cremona, senza accompagnarli a una contestualizzazione storica? Il fatto che si ragioni in termini di "pentimenti e vergogna" piuttosto che di penetrazione culturale non fa che confermare i dubbi circa la totale ignoranza, da parte dei curatori, del dibattito storiografico in materia. Un dibattito che è in corso ormai da moltissimi anni e poggia su ampie e solide basi. Ma torniamo alla mostra. Altro che esposizione improntata all'"iperfilologia" e protesa a "riabilitare" una manifestazione artistica vittima di decenni di "pregiudizio"! Dopo aver percorso l'allestimento nella crescente ansia di poter trovare le chiavi di lettura dei materiali esposti, il visitatore era colto dallo sbigottimento e dalla certezza che se avesse voluto sapere qualche cosa in più, dopo aver già pagato un più che congruo prezzo di biglietto di ingresso, era costretto ad acquistare anche il catalogo e scoprire che, a sua volta, anche quest'ultimo rappresentava l'ennesimo caso a sé. Anche qui infatti la storia sembrava non essere di casa.

\section{Dove è finita l'interpretazione storiografica?}

Facciamo un esempio. Abbiamo citato prima la cosiddetta "Marcia della giovinezza" del 1940. Era ovvio che nel contesto di un premio fortemente connotato sul piano politico come il "Premio Cremona" essa avrebbe dovuto lasciar traccia, tanto più che il "tema" del premio fissato per il 1941 fu "La Gioventù italiana del littorio". E in quell'anno Carlo Prada presentò un dipinto dedicato alla marcia. La tela fu inghiottita dalle vicende della guerra e oggi è dichiarata "dispersa". Ebbene, il catalogo se ne occupa e ne riproduce una immagine in bianco e nero tratta dal catalogo del premio, ma si guarda bene dal creare un raccordo con l'avvenimento dell'estate 1940. Bona infatti scrive: "il quadro oggi ci sembra più degno della prima pagina di un settimanale illustrato ma, all'epoca, doveva apparire come un virile richiamo 'alla soda e semplice chiarezza, e alla vita' avendo come suo fondamento quell'arte vera che Ojetti raccomandava”.[8] Evidentemente chi scrive ignora l'avvenimento e ha scambiato il titolo per il riferimento, con un esercizio di retorica tipico dell'epoca, alle attività ginniche della GIL o a uno dei canti fascisti più diffusi. Non è l'unica imprecisione di questo genere. Citeremo un secondo caso forse ancor più emblematico, a nostro avviso, di quella storia "volutamente rimossa" che sembra illuminare l'intera mostra. Si tratta della "natura" del gemellaggio Cremona- Hannover che si sviluppa fin dai primi passi del "Premio Cremona". I testi consegnati al catalogo e quelli diffusi per la promozione dell'esposizione ne parlano ampiamente ma nella forma dell'iniziativa fortemente voluta da Farinacci senza mai entrare nel merito del contesto storico che la rende possibile, cioè l'accordo internazionale italo-tedesco per consentire l'impiego di manodopera italiana nelle fabbriche e nelle campagne del Reich. L'accordo, che rappresenterà una sorta di modello anche per la riorganizzazione del mercato del lavoro nell'Europa del dopoguerra, è stato ampiamente studiato sia in Germania, sia in Italia da vari storici (per fare qualche nome: Cesare Bermani, Sergio Bologna, Ralf Lang, Brunello Mantelli, Herbert Ulrich), ricorrendo oltretutto a metodologie di ricerca d'avanguardia, come l'impiego di sistematiche campagne di raccolta di fonti orali. Ebbene di tutto questo interessantissimo percorso di ricerca storiografica non vi è traccia alcuna nel catalogo. Qui anzi si cerca di mostrare il preteso carattere fortemente ludico dell'iniziativa, con una immagine fotografica a doppia pagina che ritrae un gruppo di Balilla e Giovani italiane che visita un gruppo di soldati tedeschi feriti al fronte spacciata per una istantanea che avrebbe ritratto l'incontro tra giovani della gioventù hitleriana e un gruppo di lavoratori italiani originari di Cremona.[9]

\section{Farinacci, chi era costui?}

A proposito di queste imperdonabili "smemoratezze", come non sottolineare che anche per appagare una minima curiosità su Farinacci era necessario chiudere il volume e andare a cercare altrove le informazioni? Farinacci era ripetutamente citato in vari saggi ma, nonostante ciò, restava un soggetto assolutamente, e spesso volutamente, non approfondito, per quanto sia indiscutibile il suo ruolo di rappresentante di punta del radicalismo fascista: razzista, antisemita e filo-nazista. Farinacci dominò per un ventennio a Cremona tutta la vita economica, politica e culturale della città 
e del territorio. Inoltre, a livello nazionale, riuscì a porsi a capo di una vastissima clientela imprenditoriale capace di condizionare in modo significativo l'intero sistema affaristico italiano. Il "Premio Cremona" nasce in questo contesto cultural-politico e fu organico al percorso politico incarnato da Farinacci. Lo ammette con chiarezza lo stesso Bona fin dalle prime pagine del suo precedente saggio dedicato al premio: "se è vero che il giudizio sul clima nel quale è maturato il Premio Cremona ha per troppo tempo oscurato i fatti artistici, è altrettanto vero che considerare questi ultimi svincolati dal loro contesto storico e dalle specifiche circostanze, ideologiche e materiali, nelle quali si sono concretizzati, significa di fatto non comprenderli fino in fondo e, ancora una volta svilirli, seppur nell'ansia di rivalutarli".[10] Peccato però che alle giuste osservazioni non siano, già allora, seguiti i fatti concreti. Ma ritorniamo ancora per un attimo a Farinacci. Non certo per volontà dei promotori della mostra Il regime dell'arte, ma per una casuale concomitanza di circostanze, lo stesso palazzo che ospita i Civici musei e l'Ala Ponzone, ove era stata allestita l'esposizione dei materiali del "Premio Cremona", è anche la sede dell'Archivio di Stato. Negli spazi dell'Archivio, precedendo di alcuni mesi la mostra, era stata inaugurata l'esposizione documentaria Si faccia un articolo di fondo... 'Il Regime Fascista', Farinacci e il Ventennio a Cremona. La mostra proponeva un'ampia selezione di documenti provenienti dal fondo Renzo Bacchetta conservato presso l'Archivio, all'interno del quale è stata trovata una parte dell'archivio del giornale "Il Regime Fascista". Il quotidiano fondato da Roberto Farinacci nel 1922 non era una delle tante pubblicazioni periodiche di partito. Non a caso la primitiva testata, "Cremona nuova", era stata modificata d'autorità direttamente da Mussolini, che aveva argomentato con Farinacci la scelta con queste parole: "il tuo giornale sarà un'arma del Regime e per il Regime un valido strumento di battaglie e di fede". Il foglio si ritrovava nelle edicole di tutt'Italia, "regolarmente fino a Napoli e anche all'estero: era un quotidiano nazionale". Inoltre, la linea editoriale "volta a volta ha rispecchiato, avversato, anticipato o condiviso la politica nazionale. Una delle più significative anticipazioni/condivisioni è stata quella relativa alle leggi razziali del '38, per cui il giornale di Farinacci si distinse per tempistiche e temi trattati'[11].

\section{Farinacci nei documenti}

L'esposizione dell'Archivio di Stato di Cremona era corredata da ampi testi illustrativi che consentivano al visitatore un esauriente e documentato incontro con la figura di Roberto Farinacci. L'incontro avveniva, questo non si può tacere, con una grande fatica. trattandosi di una sorta di continuum fatto di documenti scritti, ampie didascalie, accurati testi esplicativi più adatti a un libro che ad una mostra. L'attenzione a restituire un quadro storiografico completo e aggiornato anche alla luce dei più recenti contributi della ricerca intorno a questo personaggio era più che evidente anche dalle pagine del catalogo che consentiva al visitatore di disporre della documentazione esposta nelle vetrine, ritrascritta e spesso riprodotta in fac-simile. Con grande generosità nei confronti del visitatore dell'altra mostra e delle palesi lacune storiche di questa, l'Archivio di Stato scelse di prorogare la chiusura della sua esposizione garantendo così una formidabile e indispensabile integrazione di contenuti e di informazioni storiografiche. Fu decisione non intenzionale e ancor meno di contrapposizione, anche perché venne annunciata quando ancora la linea della mostra Il Regime dell'Arte non era conosciuta, sta di fatto che le circostanze e il caso devono aver indotto molti a pensare che, ci si creda o meno, spesso occorra ringraziare manzonianamente la provvidenza.

\section{Note:}

[1] G. Brusini, Quando le canzoni parlano di noi e non solo di noi, in Noi ... non erano solo canzonette. 1958-1982, Milano, Skira, 2019, p. 19. 
[2] G. De Luna, Lacrime e sorrisi, in Noi...non erano solo canzonette, 1958-1982, Milano, Skira, 2019 , p. 21.

[3] Pieghevole di presentazione della mostra, 2018, s.i.t.

[4] Ufficio stampa SEC Spa, Il Regime dell'Arte, comunicato stampa, s.i.d. (https://contemplazioni.it/materiali-stampa/ cons. il 25 sett. 2018).

[5] V. Sgarbi, La critica contro l'arte. Fuori il Premio Cremona, in Il Regime dell'Arte. Premio Cremona 1939-1941, sil, Contemplazioni, 2018, p. 26.

[6] Sgarbi, 2018, p. 18.

[7] R. Bona, Opere in mostra, in Il Regime dell'Arte, Premio Cremona 1939-1941, sil, Contemplazioni, 2018, p. 202.

[8] R. Bona, Ragioni, difficoltà e limiti di una mostra, in Il Regime dell'Arte, Premio Cremona 1939-1941, sil, Contemplazioni, 2018, p. 93.

[9] C. Regin C., L'asse Cremona-Hannover: un gemellaggio dimenticato, in Il Regime dell'Arte, cit., pp. 56-57.

[10] R. Bona, Il Premio Cremona (1939-1941). Opere e protagonisti, Piacenza, Scritture, 2016, p. 8

[11] S. Campagnolo, Introduzione, in Si faccia un articolo di fondo... 'Il Regime Fascista', Farinacci e il Ventennio a Cremona, Cremona, Biblioteca Statale di Cremona, 2018, p. 11. 